\title{
VISIONES SOBRE RIESGO, NATURALEZA Y DESARROLLO LA PRODUCCIÓN DE PASTA DE CELULOSA EN URUGUAY
}

\author{
Valentina Pereyra Ceretta
}

\section{Resumen}

El artículo propone una aproximación al estudio de la instalación de las plantas de celulosa en Uruguay haciendo hincapié en las valoraciones que diferentes actores sociales realizan sobre este sector productivo y su vinculación con el ambiente y la naturaleza. En este sentido, se busca caracterizar los modelos de desarrollo implícitos en sus discursos para reconocer tensiones y similitudes. Se pretende avanzar en el análisis de la construcción social del riesgo, profundizando en la selección realizada por los actores y en cómo los enfrentan. Para esto, se incorporan los aportes de la literatura crítica latinoamericana y las teorías sobre relaciones sociedad-naturaleza, con el fin de analizar los discursos en estrecha relación con las nociones de desarrollo y ambiente que manejan los actores.

Palabras clave: naturaleza, desarrollo, riesgos, celulosa, extractivismo.

\begin{abstract}
Visions on the risk, nature and development. The pulp production in Uruguay

This article aims to introduce the study of the construction of pulp mills in Uruguay, placing a focus on the various social actors' assessments of this industry and the actors' relationship with the environment and nature. The purpose is to characterize the models of development implicit in social actors' discourses, identifying tensions and similarities between them. The study seeks to further the analysis of the social construction of risk, delving into the actors' risk selection regarding the pulp mill industry and how they manage risks. Contributions from Latin-American critical theory have been incorporated into the study, looking to analyze the actors' discourses in close relation to their own notions of development and environment.
\end{abstract}

Keywords: nature, development, risks, cellulose, extractivism.

\footnotetext{
Valentina Pereyra Ceretta: Licenciada en Sociología (UDELAR) y candidata a magíster en Ciencias Agrarias, opción Ciencias Sociales, en la Facultad de Agronomía (UDELAR). Investigadora y docente en el Departamento de Sociología de la Facultad de Ciencias Sociales (UDELAR).

ORCID iD: 0000-0002-5593-4375

Email: valentinapereyra24@gmail.com
}

Recibido: 19 de mayo de 2020

Aprobado: 7 de octubre de 2020

Revista de Ciencias Sociales, DS-FCS, vol. 34, n. ${ }^{\circ}$ 48, enero-junio 2021, pp.93-114. 


\section{Introducción}

La instalación de las plantas de celulosa en Uruguay ha despertado fuertes debates y cuestionamientos y, en este sentido, hablar de producción de celulosa significa también hablar de economía, desarrollo y ambiente. El debate generado a raíz de la llegada de estos emprendimientos a nuestro país derivó en demandas de incremento de seguridad, normativas y mayores cuidados para los trabajadores y para el medioambiente. En este contexto, este trabajo propone un acercamiento a los discursos sobre riesgo, ambiente y desarrollo de diferentes actores sociales vinculados al sector.

$\mathrm{Si}$ bien los cuestionamientos en torno a estos temas son relativamente recientes en Uruguay, no es así en el continente. En los últimos años del siglo XX y principios del XXI se ha acentuado en América Latina un patrón de acumulación basado en la sobreexplotación de bienes naturales-comunes ${ }^{1}$ y en la expansión de las fronteras hacia territorios considerados hasta el momento como "improductivos". Desde la literatura crítica latinoamericana (Gudynas, 2017; Svampa, 2012; Machado Aráoz, 2014) existe consenso en que se trata de una forma de producción, denominada extractivismo, basada en la explotación intensiva de los bienes comunes, y cuyo producto se destina principalmente a la exportación con poco o ningún procesamiento industrial en el país de origen. Si bien existen diferencias en las características que adquiere el extractivismo, ${ }^{2}$ en especial en cuanto al rol del Estado y la captura y redistribución de la riqueza apropiada, es pertinente utilizar esta categoría para dar cuenta de la relación que establece este tipo de producción con la naturaleza. Gudynas (2017) señala que los extractivismos refuerzan las concepciones de la naturaleza como "agregado de recursos", es decir, parten de una perspectiva utilitarista entendiéndolos como meras mercaderías. Según el autor, esta manera de concebir a la naturaleza impide entender su

1 El concepto de bienes naturales-comunes (bienes comunes) alude a la necesidad de mantener fuera del mercado aquellos bienes que, por su carácter de patrimonio natural, social o cultural, pertenecen al ámbito de la comunidad y poseen un valor que rebasa cualquier precio (Svampa, 2012). Se trata de romper con la noción de naturaleza como recurso para ser utilizado y manipulado.

2 Este enfoque presenta dos tipologías principales en las cuales podrían incluirse los distintos países de la región: por una parte, el extractivismo propiamente dicho, ligado a los gobiernos neoliberales; por la otra, el llamado "neoextractivismo", ligado a los gobiernos de tinte progresista. Ver en este sentido: Gudynas (2013) y Pérez Trento (2020). 
organicidad y reconocer en ella otros valores, no utilitarios, y minimiza los impactos ambientales.

Estas configuraciones productivas dejan sus huellas en el ambiente y los territorios: desforestación y pérdida de biodiversidad, destrucción de ecosistemas, erosión de los suelos y contaminación de fuentes de agua, pérdida de reservas energéticas, problemas de salud y contaminación, afectación de la seguridad hídrica y alimentaria, degradación de las dietas e incremento incesante de desplazados y refugiados ambientales, son algunas de las actuales afecciones (Bartra, 2009).

En esta coyuntura, Uruguay no representa una excepción. Carámbula y Piñeiro (2006) concluyen que en los últimos años del siglo XX se gestaron importantes transformaciones en la producción y la economía uruguayas, fundamentalmente la reconfiguración del paradigma agrícola hacia un modelo de agronegocio. El agronegocio como nuevo modelo de producción se instala en el país de la mano de las grandes corporaciones internacionales relacionadas con la producción de granos y la forestación. La presencia más evidente del ingreso de transnacionales en el agro se dio sobre todo con la expansión de las empresas forestales. Si bien en sus inicios la producción forestal con destino industrial tuvo una alta presencia nacional, a fines de la década del noventa se inició un proceso de extranjerización de las plantaciones que contó con las empresas Weyerhaeuser, FOSA-BOTNIA y ENCE como las principales protagonistas (Florit, 2013).

Juncal y Fernández (2013) proponen dividir la evolución del complejo forestal-celulósico en Uruguay en tres etapas: una primera fase de instalación, que abarcaría el período comprendido entre la promulgación de la Ley n. ${ }^{\circ} 15.939$ (1987) y el año 2007, en el cual comienza a operar la primera planta de pasta de celulosa Botnia (hoy UPM), próxima a la localidad de Fray Bentos, en el departamento de Río Negro, sobre el río Uruguay. Una segunda fase está signada por la primera planta ya en funcionamiento y por la instalación de la segunda planta (Montes del Plata), próxima a la localidad de Conchillas, en el departamento de Colonia, sobre el Río de la Plata. Por último, la tercera etapa podría caracterizarse como de expansión y conlleva la instalación de una tercera planta de procesamiento de pulpa de celulosa a ser ubicada en la localidad Pueblo Centenario, en el límite departamental entre Durazno y Tacuarembó, sobre el río Negro.

De esta forma, las industrias maderera y papelera en Uruguay han crecido aceleradamente en los últimos doce años, duplicando el porcentaje que ocupan en la composición del producto interno bruto (PIB) en relación, por ejemplo, con 1998 (Tommasino, et al., 2016). En el informe de Uruguay XXI (2016), con base en datos del Banco Central del Uruguay (BCU), se señala que el PIB de la fase primaria muestra una trayectoria creciente, exhibiendo una tasa promedio de crecimiento de $8,7 \%$ anual en la última década. Por su parte, el valor agregado en la fase industrial también muestra una trayectoria 
fuertemente creciente (sobre todo a partir del comienzo de las actividades de la planta de celulosa de UPM, sobre finales de 2007, y de Montes del Plata, a mediados de 2014), manteniendo un crecimiento promedio anual de $16,2 \%$ en la última década (Uruguay XXI, 2016).

En cuanto a los riesgos asociados al sector, Nión y Pereyra (2019) dan cuenta de que diversos actores relacionados con él identifican riesgos que responden a diferentes problemáticas. Las autoras los agrupan en seis categorías: laborales (uso intensivo de químicos, no uso de elementos de protección personal, atrapamiento, ruido, estrés, sedentarismo, trabajo confinado); productivos (dinámicas durante las paradas de mantenimiento, clausuras, falta de insumos para la producción); ecológicos (contaminación, uso extractivo de bienes comunes); socioeconómicos (desplazamiento de mano de obra, nuevas dinámicas sociales, subcontratos); comunitarios (repercusiones en la salud humana, impacto en las dinámicas comunitarias y sus identidades, conflictos socioambientales, muy especialmente, problemáticas relacionadas con la explotación sexual, los accidentes de tránsito y el aumento del alcoholismo) e institucionales o colectivos (falta de trabajo en conjunto entre actores, falta de espacios para la participación en la toma de decisiones). ${ }^{3}$

Este trabajo se propone aportar a los debates sobre los riesgos asociados a la producción de celulosa a partir del análisis de los discursos de tres actores vinculados al sector: organismos estatales, sindicatos de las empresas de producción celulosa y colectivos ambientalistas. Específicamente, ahonda en las valoraciones que estos actores hacen sobre el sector productivo en vinculación con el ambiente, la naturaleza y las concepciones de desarrollo. Con este objetivo, primero se presentan algunos conceptos teóricos sobre riesgo y relaciones sociedad-naturaleza que dan base al trabajo; en el apartado segundo, se explicita la metodología de la investigación; el tercero, se corresponde con el análisis y el diálogo de los discursos de los actores desde las bases teóricas antes expuestas; y en el cuarto y último apartado se presentan las conclusiones y reflexiones finales.

\section{Valoraciones sobre ambiente/naturaleza y riesgo}

\section{Relaciones entre sociedad y ambiente/naturaleza}

Desde comienzos del siglo XXI, en diversos ámbitos de la sociedad uruguaya se observa una creciente importancia asignada a la temática ambiental, especialmente a la urgencia de encontrar soluciones a los problemas ambientales

3 Durante el proceso, es importante diferenciar dos etapas: la construcción y la puesta en funcionamiento. Muchos de los riesgos asociados a lo comunitario se enmarcan en la etapa de construcción. Para profundizar en los riesgos asociados a la producción de celulosa, ver: Nión y Pereyra (2019). 
emergentes. En esta línea, Gazzano y Achkar (2013) señalan que la irrupción de la temática ambiental en los ámbitos sociales, culturales, científicos y políticos constituye un nuevo factor cuestionador que coloca en debate conceptos como desarrollo, tecnología y ciencia.

En el ámbito mundial, hace varias décadas se comenzó a señalar que la crisis ambiental es en realidad una crisis civilizatoria, en la que no se puede separar la degradación de la dimensión físico-biológica del deterioro social (Leff, 1998). Según Bartra (2009), el problema ambiental se presenta en primera instancia desde un contexto biofísico y cuando se lo intenta unir, para su interpretación, a la dimensión económica y social, puede ser reformulado como "crisis civilizatoria".

Beck (1985) declaraba la emergencia y constitución de la "sociedad del riesgo", refiriendo con ello a "una civilización que se pone en peligro a sí misma". Este planteo apuntaba a resaltar una serie de cambios decisivos de la problemática ecológica de la modernidad. En esta época se asume que los "beneficios del progreso científico-técnico" se ven crecientemente eclipsados por la producción de peligros y de riesgos masivos y de vasto alcance. A su vez, estos riesgos no son repartidos de manera equitativa entre la población, y son por lo general las poblaciones más vulnerables aquellas que también sufren las mayores consecuencias negativas ambientales y sociales.

En este contexto, resulta interesante introducirnos en los debates sobre las relaciones sociedad/naturaleza a partir de los aportes de Schwarz y Thompson (1990). Estos autores analizan que las distintas visiones sobre la naturaleza derivan de visiones distintivas de la sociedad. En otras palabras, lo anterior implica que los ideales de naturaleza se apoyan en un conjunto de valores y creencias. En el caso de este trabajo, las relaciones que se establecen entre sociedad y naturaleza dan base a los diferentes discursos y evaluaciones que los actores hacen sobre los modelos de desarrollo, sus riesgos y, en definitiva, de ser, estar y habitar los territorios.

Las propuestas sobre las tipologías de naturaleza y política ambiental de Schwarz y Thompson (1990), que son retomadas por Douglas (1996), indican que existen diferencias sistemáticas en el modo en que los individuos perciben los riesgos ambientales. Estos autores identifican cuatro mitos distintivos de la naturaleza; cada uno de ellos es una explicación del mundo que justifica el estilo de vida con el que está comprometido su expositor y este compromiso no es una intención privada, sino que es parte de la cultura a la cual el expositor eligió adherir:

- Nature benign o "la naturaleza robusta": en esta visión, la naturaleza es suficientemente resiliente ante cualquier intervención humana. Se corresponde con un modo de organización social "individualista", donde cada quien persigue sus intereses sin tener en cuenta el bien colectivo (Schwarz y Thompson, 1990). Según Douglas (1996), esta 
versión justificaría el avance de los modelos productivos extractivistas sin importar las advertencias sobre contaminación o problemáticas ambientales.

- Perverse/tolerant o "la naturaleza necesita estructura": desde esta perspectiva, se entiende que la naturaleza es tolerante, robusta y estable, pero solo dentro de ciertos límites. Los seres humanos pueden intervenir la naturaleza sin alterar el balance, pero solo hasta cierto punto, pasado el cual el daño puede ser irreversible. Desde esta visión, se plantea que la sociedad debe establecer reglas que prevengan que ese punto sea sobrepasado. Esta perspectiva se suele asociar con la idea de jerarquía, ya que sugiere un control social de comportamiento centralizado por una autoridad, justificando también la instauración de controles y la planificación de proyectos.

- Nature capricious o "la naturaleza es impredecible": esta visión defiende el carácter impredecible de la naturaleza y la idea de que nadie puede saber el curso de los acontecimientos (Douglas, 1996). Se afirma desde esta posición que no tiene sentido tratar de controlar al medioambiente, dado que los cambios van a ocurrir con o sin la acción humana. Un ejemplo de esto se encuentra en las discusiones sobre el cambio climático o las visiones fatalistas.

- Nature ephemeral o "la naturaleza bajo presión": aquí se advierte que la naturaleza es susceptible de sufrir cambios permanentes. Propone una actitud cuidadosa hacia el ambiente y las posibles consecuencias de la intervención humana. Esta posición ha entrado en conflicto fundamentalmente con la política de los empresarios y con los jerarquistas.

En este punto, es interesante retomar los aportes de Svampa y Viale (2014), quienes, con una mirada histórica, señalan que desde los inicios de la modernidad la relación de la humanidad con la naturaleza ha sufrido una alteración radical que se ha ido profundizando hasta el presente: el ser humano primero se desconectó de la naturaleza, la concibió como objeto de conocimiento, luego como objeto de dominación y, finalmente, como una mera mercancía.

En el ámbito latinoamericano, Quijano (1992) vincula los procesos extractivistas que mencionábamos en el apartado anterior con un proceso de completa reconfiguración de la colonialidad ${ }^{4}$ del poder, reconociendo la aceleración y la profundización de la tendencia a la concentración del control por el poder desarrollista que alienta y alimenta la voracidad extractiva. Uno de los elementos de esta relación es el radical dualismo cartesiano que

4 Colonialidad del poder fue la expresión utilizada por Quijano (1992) para caracterizar un patrón de dominación global propio del sistema-mundo moderno/capitalista originado con el colonialismo europeo a principios del siglo XVI. 
separa naturaleza y razón; de aquí que una de las imágenes más comunes en este sentido es que la explotación de la naturaleza no requiere justificación alguna, lo que expresa cabalmente la ética productivista. Estas ideas también podrían ser relacionadas con la tipología de naturaleza robusta de los citados Schwarz, Thompson y Douglas. También autores como Sack (1986) denuncian que en función de una mirada productivista y eficientista de la naturaleza se alienta la descalificación de otras lógicas de valorización, y así los territorios escogidos por el capital pasan a ser considerados como "socialmente vaciables" o "sacrificables".

A partir de lo anterior, Machado Aráoz (2014) plantea la necesidad de tener en cuenta las valoraciones sobre los territorios desde la subjetividad de los actores:

"Ver y sentir las agresiones a los territorios como agresiones a los propios cuerpos es ciertamente una cuestión 'subjetiva'. Eminentemente subjetiva. No sentir nada ante los 'impactos' mineros es algo también 'subjetivo'. 'Subjetividad' y 'objetividad' remiten, en definitiva, a la forma política en que se procesan los sentimientos humanos - personales y colectivos- en los discursos y las prácticas. Allí donde se define la base motivacional de los proyectos civilizatorios." (Machado Aráoz, 2014, p. 67).

Finalmente, resulta importante incorporar la perspectiva de Ingold (2000) para reflexionar sobre esta idea de naturaleza. El autor propone la perspectiva del habitar, y habla de ambiente - no de naturaleza-, al cual define como una zona de interpenetración continua. El ambiente no es una naturaleza exterior, sino el producto parcial siempre en construcción que surge de la interacción entre la vida humana y la no humana. Si bien este es un debate profundo, que excede las pretensiones de este artículo, interesa nombrarlo aquí porque da cuenta también de una forma de vincularse y de entender los conceptos de ambiente/naturaleza y la importancia de su interrelación.

\section{¿Cómo y quiénes definen los riesgos?}

Las teorías del riesgo señalan que las actividades de producción se despliegan, en la actualidad, en contextos de creciente incertidumbre e indeterminación, marcando la existencia de riesgos propios de cada proceso y tarea. Con base en esto, Douglas (1996) señala que los individuos tienden a tener un fuerte sentido de inmunidad subjetiva. Con esto quiere decir que en las actividades que nos son familiares existe la tendencia a minimizar la probabilidad de malos resultados, es decir, se subestiman aquellos riesgos que se consideran controlados. En el otro extremo de la escala de posibilidades, se tiende a restar importancia a los peligros más infrecuentes y de baja probabilidad.

Lo anterior nos lleva a señalar que no existe el riesgo cero, lo que existe son diferentes umbrales aceptables de riesgo, por ejemplo, según se participe en el 
riesgo como portador de decisiones o como afectado por ellas. Las definiciones de los riesgos y cómo afrontarlos difícilmente generen consensos absolutos, aspecto que veremos claramente reflejado en los conflictos y disputas entre los diferentes actores por los impactos de la producción de celulosa.

La existencia de actitudes y comportamientos con relación al riesgo define culturas y umbrales aceptables, como producto de los valores insertos en una estructura de poder social determinada y construidos socialmente a partir de creencias y normas sociales (Pucci, Levin, Trajtenberg y Bianchi, 2006). A su vez, las decisiones sobre el riesgo implican un vínculo con el tiempo, ya que son decisiones elaboradas en el presente pero proyectando sobre acontecimientos que podrían suceder en el futuro.

En relación con las problemáticas ambientales, Douglas (1996) señala que los peligros o riesgos son seleccionados por las personas culturalmente, es decir, no todos son efectivamente peligros/riesgos, solo algunos. Además, los procesos culturales que seleccionan los tipos de peligro a atender funcionan mediante procedimientos que asignan responsabilidades. El conocimiento sobre los riesgos pasa a ser histórico, local y cuestionado constantemente; su naturaleza y control están sujetos socialmente a disputas. En este sentido, también se pone en juego la legitimidad de la atención a esos riesgos, quiénes pueden y quiénes deben atenderlos son cuestiones en constante debate.

A partir de lo planteado, este trabajo analiza los discursos sobre riesgo de los actores relacionados con la producción de celulosa, en vinculación con las visiones sobre naturaleza/ambiente y desarrollo que estos manejan, como parte también de una construcción social. La relación que los actores construyen (implícita o explícitamente) con la naturaleza/ambiente es un elemento clave para comprender la definición y la selección de riesgos que ellos hagan y cómo deben ser manejados y afrontados esos riesgos, así como para entender sus visiones sobre el modelo en cuestión.

\section{Metodología y recolección de datos}

El trabajo forma parte de la investigación "Organización del trabajo y gestión del riesgo en la producción de pasta de celulosa", 5 llevada a cabo durante 2017 y 2018. En la investigación se utilizó una aproximación cualitativa para enmarcar la producción de pasta de celulosa en el contexto de los actores sociales $^{6}$ vinculados al sector, sus definiciones de los riesgos y los ámbitos de atención y legitimidad del conocimiento para atenderlos o controlarlos.

$5 \quad$ Financiado por la Comisión Sectorial de Investigación Científica (CSIC) en el llamado I+D 2017 y ejecutado por el Departamento de Sociología de Facultad de Ciencias Sociales (DSFCS), UDELAR.

6 Son concebidos como grupos, organizaciones o personas representativas de un dominio de saber y poder en torno a los riesgos. Los actores sociales son definidos en función de un aspecto específico de la realidad analizada. 
En primer lugar, se realizó un mapeo de actores sociales, de modo de poder representar las diferentes visiones en torno a la gestión del riesgo en la producción de pasta de celulosa. El mapeo es una herramienta que permite no solo listar posibles actores vinculados a un sector, sino también identificar roles adjudicados, distribuciones de poder y legitimidad. El listado de actores sociales se realizó entre investigadores y participantes según su vínculo con el sector y la legitimidad de su participación (y su saber) en la definición de los riesgos o su atención. Una vez generado el listado, espontáneamente, en entrevista o taller, se les consultó específicamente por actores que no habían sido mencionados, de forma que también se relevó por qué algunos no surgieron o no fueron tenidos en cuenta.

La muestra se basó en la teoría y los antecedentes e integró casos a partir de una selección por juicio, pero también por las dimensiones que fueron surgiendo como relevantes en el transcurso del trabajo de campo. En relación con las empresas, la muestra se compuso por las dos empresas del sector y, dentro de ellas, un segundo nivel relevó información brindada por el personal, tomando en cuenta las diferentes jerarquías, tanto de las áreas de gestión (recursos humanos, seguridad, responsabilidad social, producción, salud) como de las áreas operativas (madera, producción de chips, producción de químicos, cocción, blanqueo, formación pulpa blanqueada, proceso de secado, generación de vapor y recuperación, tratamiento de agua y efluentes, laboratorio) (ver Tabla 1).

En términos de las técnicas de recolección de información, por un lado, se realizaron entrevistas semiestructuradas, contemplando los ejes analíticos del marco teórico, pero conservando la libertad de profundizar en aspectos que pudieran emerger en las entrevistas mismas. En total, se realizaron 31 entrevistas semiestructuradas (individuales y grupales).

También se realizaron talleres en la metodología de paneles de expertos. Estos paneles pueden definirse como grupos de especialistas en al menos uno de los aspectos que hacen a la temática. En este caso, por "experto" no se hizo referencia a personas con un determinado conocimiento especializado, sino en el sentido de informante calificado. Se realizaron dos paneles, uno en 2017 y otro en 2018, a los que acudieron, en cada uno, alrededor de 15 participantes, y se trabajó en grupos a partir de una consigna. En el segundo panel, se presentaron reflexiones preliminares sobre el trabajo de campo realizado hasta el momento, en un esfuerzo por comenzar a debatir los resultados y reflexiones en curso.

Asimismo, se realizó observación en instancias de discusión sobre el sector: paneles, conferencias y mesas redondas, y se realizaron observaciones no participantes semiestructuradas de las instalaciones y procesos productivos dentro de las empresas.

Desde el punto de vista cuantitativo, se analizaron datos secundarios para caracterizar el sector: características productivas, de la fuerza de trabajo, 
organización y proceso de trabajo, relaciones laborales, normativa, entre otros. Por último, se propuso el análisis de diversos materiales audiovisuales y prensa, mediante el cual también se relevaron posturas y visiones de los diferentes actores.

Como instancia de socialización de resultados, se llevó a cabo una actividad de intercambio y validación con actores sectoriales que participaron en las diferentes instancias de entrevistas y talleres, sobre la base de resultados preliminares de la investigación.

Tabla 1. Actores relevados por la investigación

\begin{tabular}{cc}
\hline Clasificación & Actores participantes en paneles o entrevistas? \\
\hline DINAMA, MVOTMA, Área Control y Desempeño Ambiental \\
DINAMA, MVOTMA, Área de Impacto Ambiental \\
DINAMA, MVOTMA, Dirección \\
MSP, Dirección de Salud Ambiental y Ocupacional \\
MSP, asesora \\
IGTSS, MTSS, Dirección
\end{tabular}

Fuente: Elaboración propia.

7 Referencia de siglas: ver Tabla 2 en anexo. 


\section{Riesgos y actores en la producción de celulosa}

De los diversos actores relevados en la investigación, este artículo se centra en los referentes estatales, en particular de las áreas salud y ambiente, los referentes sindicales de las empresas y los colectivos ambientalistas. En relación con esta elección, se entiende pertinente realizar dos aclaraciones.

Por un lado, los entrevistados representantes de organizaciones/ instituciones, como es el caso de los tres incluidos en este trabajo, fueron buscados en calidad de representes de la organización/institución a la cual pertenecían, por lo que se entiende que su postura refleja el discurso de la organización que representan. Dentro de los actores estatales, en este artículo, se eligió trabajar con la Dirección Nacional de Medio Ambiente (DINAMA) como institución responsable del control ambiental en el territorio uruguayo y con el Ministerio de Salud Pública (MSP) por su cometido de cuidado de la salud de la población.

Por otro lado, en relación con la pertinencia de focalizar en estos actores, se entiende que aportan tres visiones particulares del sector: la estatal, desde su legitimidad para controlar y gestionar tanto el sector como los riesgos, los sindicatos de trabajadores, por los supuestos beneficios y el contacto directo con los posibles riesgos, $\mathrm{y}$, finalmente, los ambientalistas, como portadores de la visión crítica. Los tres actores, además, aportaron visiones con diferentes grados de proximidad con la problemática: general, desde dentro y desde fuera, respectivamente. Es interesante poner en juego estas tres miradas que aparentemente tienen intereses y preocupaciones diferentes, pero que, a su vez, pueden llegar a compartir concepciones de naturaleza y desarrollo.

\section{Riesgo, naturaleza y desarrollo desde los actores estatales}

En primer lugar, es interesante destacar que tanto la DINAMA como el MSP identificaron espontáneamente la posibilidad de ocurrencia de riesgos ambientales asociados al uso de "recursos naturales", las posibles implicancias para el medioambiente y, tangencialmente, su influencia en la salud humana. El uso de la palabra "recurso" no es arbitrario, ya que desde la perspectiva de otros actores, como los ambientalistas, se optó por usar la expresión "bienes naturales", en un esfuerzo explícito por incorporarla a sus discursos como parte de una deconstrucción política de la naturaleza como recurso.

En el sector salud, los entrevistados hicieron énfasis en riesgos asociados a lo comunitario y social, especialmente en la etapa de la construcción de las plantas de producción de celulosa (aumento de la explotación sexual, incluso infantil, aumento de la violencia, accidentes de tránsito, alcoholismo, etcétera). Estos tipos de riesgos fueron asociados no tanto a la producción de celulosa en sí misma, sino a la concentración de operarios y a los cambios en la dinámica de los territorios. A su entender, su accionar debería estar más 
centrado en esta etapa de la gestión de los riesgos en tratar de controlar y acompañar los cambios en las dinámicas de las poblaciones a las que llega este tipo de emprendimientos.

En relación con el funcionamiento de las plantas, la identificación de riesgos no fue tan clara: si bien se insinuó la posibilidad de problemas ambientales, estos fueron identificados como problemáticas de la que deberían ocuparse otras instituciones. Esta delegación de la gestión del riesgo operaría más aún para el caso de los riesgos ambientales, donde, si bien se identificó la posibilidad de contaminación provocada por este tipo de producción, se notó una confianza (y delegación) en el control ejercido por parte de otros actores estatales.

En cuanto a los actores estatales vinculados con el ambiente (DINAMA), la identificación primaria de riesgos fue aún menos clara, y si bien se identificaron algunos riesgos de tipo ambiental, se percibió un esfuerzo continuo por evitar profundizar en el tema, llegando incluso a tratar de evitar el uso de la palabra "riesgo". Asimismo, realizaron un gran esfuerzo por enfatizar la existencia de controles ambientales y la normativa desplegada.

Al referirse a los posibles efectos sobre el ambiente, los referentes de este sector hicieron importante hincapié en los controles técnicos disponibles para regular las cargas y vertidos al río (vale señalar que las preocupaciones ambientales se centraron mayormente en los efectos de esta industria en los ríos y el agua). Un argumento frecuentemente esgrimido fue el uso de las "mejores tecnologías disponibles" (best available techniques [BAT]) y el acatamiento de los estándares internacionales de seguridad. Asimismo, la preocupación principal de estos representantes fue asegurar a los entrevistadores la existencia de un monitoreo continuo y de un sistema eficiente que permite la recolección de información sobre emisiones en directo.

También se observó en los discursos de los entrevistados estatales vinculados con el ambiente un continuo esfuerzo por comparar esta actividad con otras actividades industriales, sacando el foco de los problemas ambientales que esta en particular genera. El ejercicio de la comparación también fue llevando al plano de la vida cotidiana de las personas, cotejando estas actividades de producción con los efectos que generan los residuos de los individuos en sus hogares.

Estos argumentos fueron fuertemente debatidos por los colectivos y organizaciones no gubernamentales (ONG) ambientalistas, que se esforzaron por demostrar el estado preocupante de los ríos de Uruguay en cuanto a contaminación. Por su parte, los actores relacionados con la salud también demostraron preocupación por la capacidad de "resistir" de los ríos en el país ante la instalación de una nueva planta.

Ahora bien, en líneas generales, en ambos actores (ambiente y salud) se identifica un discurso que tiende a compartimentar la responsabilidad de la atención de los riesgos, así como la injerencia sobre ellos: seguridad laboral 
incumbe al Ministerio de Trabajo y Seguridad Social (MTSS), salud laboral o salud de la comunidad al MSP y los posibles impactos ambientales a la DINAMA.

Asimismo, ambos actores coincidieron en la identificación del rol primordial de los controles estatales para el correcto funcionamiento y para minimizar los riesgos. La idea de controlabilidad en estos actores se encuentra muy vinculada al concepto de umbrales de riesgo que se desarrolló en el apartado teórico. Sobre esta idea de controlabilidad de los riesgos es pertinente realizar dos reflexiones.

Por un lado, la identificación de riesgos se encuentra estrechamente ligada a la noción de controlabilidad y manejo mediante las normativas y el uso de las BAT. Es decir, existe un discurso compartido en torno a que estas empresas aumentaron los estándares de seguridad ambiental y laboral en el país. A partir de esto, se podría señalar que estos factores actúan como moduladores en la percepción de los riesgos, situándolos en una órbita de controlabilidad y un sentimiento de seguridad y confianza sobre su manejo: son garantía de seguridad. En este sentido, la tecnología que podría ser vista como la fuente de los riesgos es considerada, en cambio, como un medio de protección.

Por otro lado, específicamente en el caso de la DINAMA, si bien los referentes reconocen que existieron ciertos problemas en los controles, reflejados en eventos de contaminación o derrames, e inclusive admiten la posibilidad de generación de efectos ambientales negativos - aspectos que podrían pensarse como factores que escapan a esa controlabilidad-, estos son entendidos como riesgos aceptables o esperables. Así, en cierta medida, vuelven a ser "controlables", dado que se encuentran dentro de los parámetros admisibles, es decir, dentro de los umbrales aceptables de riesgo.

Otro argumento que aparece frecuentemente es la idea de que es muy difícil rastrear la contaminación, porque las aguas "ya vienen contaminadas". En este argumento se percibe tanto una delegación de la problemática ambiental a otros actores como una suerte de licencia o permiso para verter cargas al río.

Pensando en los mitos sobre la naturaleza propuestos por Schwarz y Thompson (1990) y Douglas (1996), se podría asociar estos discursos a la visión de la naturaleza "fuerte y robusta, pero que necesita estructura". En esta visión, la contaminación parecería estar permitida dentro de ciertos límites, que son definidos por autoridades o jerarquías institucionales, ya que solo algunos actores tienen la legitimidad de definir los riesgos y sus umbrales. En el caso de los actores estatales, tanto la idea de umbrales aceptables de riesgo como la autodefinición de la legitimidad para atenderlos (basada en conocimiento, experiencia e institucionalidad) apareció con frecuencia en sus discursos. 
Llevando estas ideas al campo de las relaciones con el ambiente y las concepciones de desarrollo, en los discursos de los entrevistados aparece frecuentemente la vinculación entre estas empresas y el desarrollo mediante la generación de puestos de empleo y la revitalización de zonas donde las oportunidades laborales y el desarrollo económico eran escasos. En sus discursos, los beneficios de la inversión justificarían la necesidad de asumir ciertos riesgos ("cuantificables y controlados") o aceptar ciertos niveles de contaminación (umbrales aceptables de riesgo). Se observa entonces cómo en estos discursos entra en juego aquella idea de los costos sacrificiales del progreso o el desarrollo que mencionaban Sacks (1986) y Machado Aráoz (2014), según la cual argumentos como la generación de empleos y la necesidad urgente de crecimiento económico justifican la existencia de impactos ambientales a mediano y largo plazo. Estos niveles de contaminación son entendidos por los actores como parte de una ecuación que sienta su base en una visión productivista de la naturaleza.

\section{Las visiones desde los sindicatos de las empresas de celulosa}

Dado el tipo de actor al cual refiere este apartado, resulta ineludible hacer mención, aunque sea mínimamente, a los riesgos de tipo laboral. Estos actores demostraron un amplio consenso en que los riegos laborales han disminuido debido a las mejoras en las condiciones laborales del sector, en especial en relación con su símil papelero.

Asimismo, el riesgo más cotidiano, de accidentes o enfermedades individuales, ha sido sustituido por la visión del potencial del riesgo de explosión de la planta; es decir, cambia la idea de riesgo laboral por la idea de un posible riesgo industrial. El estudio realizado por Nión y Pereyra (2019), mencionado en la introducción de este trabajo, da cuenta de que el cambio en la identificación de riesgos les confiere a los trabajadores un peso mayor en la responsabilidad, dado que ellos son responsables de un posible riesgo ambiental y social de gran magnitud.

En este actor también se observó que existe una idea de estos emprendimientos como motores de desarrollo, asociada a las externalidades positivas de las empresas. Al igual que en el discurso de los actores estatales, estas empresas contribuirían al desarrollo de zonas "olvidadas" y serían oportunidades de progreso para muchos. Las externalidades negativas aparecen, según el entender de estos actores, bajo control. Como en los actores estatales, se observó que existe una gran confianza en los controles y mecanismos de seguridad de las empresas, asociado a lo cual también surgió con frecuencia el argumento de la utilización de BAT. Los controles, las BAT y la normativa existente actuarían como moduladores de percepción, haciéndolos sentir más seguros. El riesgo ambiental también aparece, a su entender, como esperable y controlado. 
Ahora bien, en sus discursos aparece una cierta preocupación por crear conciencia en los trabajadores, sobre todo en los más jóvenes o los que recién ingresan, de los riesgos que pueden conllevar los posibles descuidos o accidentes. Los entrevistados señalaron que a veces la automatización y tecnologización de las tareas es tal, que se puede perder la noción de que se está trabajando con "recursos naturales" y en el ambiente, solo a "través de máquinas, como un jueguito de computadora". De aquí es posible inferir que las características del trabajo a través de computadoras y salas de control podrían actuar como un factor de minimización en la percepción de riesgos y posibles impactos en el ambiente. Desde los sindicatos se señaló un esfuerzo por trabajar en la concientización del tipo de trabajo que se realiza y los posibles riesgos que implica.

Relacionando este discurso con las tipologías de Schwarz y Thompson (1990) y Douglas (1996), podemos suponer que este grupo también entraría dentro de la visión de la naturaleza robusta pero necesitada de una estructura. En este caso, los actores también asumen una idea de naturaleza como recurso a utilizar, pero dentro de ciertos límites marcados. Los encargados de estas delimitaciones serían las autoridades y jerarquías estatales y de la empresa.

Centrándonos en la vinculación con la naturaleza y el ambiente, al igual que en los actores estatales, predomina un discurso que naturaliza el impacto en la naturaleza y defiende la controlabilidad de los riesgos y los beneficios asociados. En este caso, también se entiende que los riesgos ambientales están bajo control tanto por la normativa desarrollada y los controles (de habilitación y funcionamiento) aplicados en ese marco como por la tecnología utilizada. Incluso se podría esbozar que en el caso de los actores sindicales las externalidades positivas son aún más notorias, dado que gran parte de la mano de obra proviene de otros rubros donde las condiciones laborales son más precarias y para quienes el trabajo en las plantas de celulosas representó un cambio cualitativo y cuantitativo importante en sus trayectorias de vida. Sin embargo, también resulta interesante señalar que a lo largo de la investigación los representantes sindicales participantes mantuvieron siempre una postura abierta al diálogo con otros actores, inclusive aquellos más críticos del sector del cual ellos forman parte.

Por último, un aspecto interesante a plantear y profundizar es la relación de estos sindicatos con los demás sectores sindicales. En los últimos períodos, estos sindicatos incipientes se vieron muy apoyados por el Sindicato Único Nacional de Trabajadores de la Construcción y Anexos (SUNCA) y la Unión Nacional de Trabajadores del Metal y Ramas Afines (UNMTRA), llegando incluso a aparecer como sindicatos "propasteras". ${ }^{8}$ Esto ha generado tensiones y posturas encontradas en la interna de movimiento sindical, donde, dentro de la misma central, conviven y se enfrentan posturas muy disimiles sobre los

8 El término pasteras refiere a las plantas de celulosa, por producir pasta de celulosa. 
modelos de desarrollo y, específicamente, sobre los beneficios o perjuicios de este tipo de emprendimientos.

\section{La visión desde los colectivos ambientalistas}

El último conjunto de actores que se aborda en este trabajo está integrado por aquellos grupos que presentan críticas a los emprendimientos vinculados con el modelo forestal-celulósico. Resulta interesante abordar las posturas de estos grupos, dado que presentan importantes diferencias con los anteriores, tanto en la identificación de riesgos como en la visión general sobre el relacionamiento de este tipo de producción con los conceptos de ambiente y desarrollo.

Los colectivos ambientalistas fueron los que más importancia les dieron a los posibles riesgos del sector, identificando una gama mucho más amplia de problemáticas ambientales asociadas. Estos actores también identificaron riesgos de diversa índole: sociales, comunitarios, políticos y, muy especialmente, ambientales y económicos.

Es importante señalar que en la identificación de riesgos, los entrevistados de este grupo no se limitaron a los efectos concretos de las plantas de celulosa sobre el ambiente, sino que abarcaron al modelo forestal-celulósico completo. A modo de ejemplo, esto implicó tomar en cuenta las consecuencias de la forestación como materia prima necesaria, los impactos del transporte de la madera y el futuro tren para la tercera planta de UPM, e incluso llegar a plantear preocupaciones que vinculan este tipo de producción con la pérdida de soberanía estatal y alimentaria de los Estados y los pueblos. Esta gama de críticas da cuenta de una visión más integral del riesgo, en el sentido de que no los compartimentan por sectores (forestación, celulosa, transporte, etcétera), sino que los entienden como parte de un mismo e indisociable fenómeno, es decir, como parte de un modelo de desarrollo. Algo similar planteaba Graña (2010) al analizar los discursos de los asambleístas de Gualeguaychú, en los que veía que "la contaminación segura es el argumento más recurrente entre los asambleístas; sin embargo, para ellos este es el aspecto más visible de una problemática global: el modelo forestal [...] que nos afecta a todos" (Graña, 2010, p. 75).

Como ya fue mencionado, estos actores son también muy críticos con los controles ambientales establecidos por las empresas e incluso de los organismos estatales, llegando a desconfiar de su efectividad y legitimidad. También señalan que los convenios para la protección del ambiente no son suficientes y, asimismo, que el argumento de la utilización de BAT no es acertado, dado que en otros países, de Europa por ejemplo, las tecnologías utilizadas son mucho menos invasivas para el medioambiente. Por último, también advierten que los impactos no son visibles a corto plazo, sino que debemos esperar sus efectos en años venideros. 
También desde este grupo se denuncia la falta de espacios para la discusión sobre la instalación de estos emprendimientos y la falta de legitimidad que se le otorga a su conocimiento. En muchas ocasiones, su discurso es invisibilizado o ridiculizado con la excusa de que no poseen el conocimiento legítimo para participar de los debates. Esto refleja una clara disputa por la legitimidad del conocimiento, la definición y atención de los riesgos, aspecto que también se observaba, en contraposición, en la autopercepción de legitimidad de los actores estatales.

Retomando los planteamientos de Villegas (2014) sobre los movimientos socioambientales en Latinoamérica, se visualiza que la resistencia en este contexto muchas veces se vuelve difícil, al ser estos actores con frecuencia ignorados y descalificados por los grandes medios de comunicación, políticos y algunos sectores del mundo académico. El autor señala que los colectivos opositores emergen como los "bárbaros" de nuestros tiempos, los que se oponen al "desarrollo" o como fracciones "fundamentalistas" que ponen trabas al progreso.

En esta línea, en los talleres y entrevistas realizados en el marco de la presente investigación, también se denunció la falta de tratamiento de la temática ambiental en el ámbito público. Esta temática no parece estar puesta sobre la mesa en muchas de las discusiones o, cuando lo está, se la coloca en un lugar secundario en relación con las necesidades de inversiones y trabajo y las promesas de desarrollo económico.

"Siempre está este discurso, por decirlo de alguna forma, de los ambientalistas versus el desarrollo [...], pero en este momento ni siquiera están los ambientalistas porque directamente el tema ambiental no está planteado. [...] La universidad salió a decir 'el río Negro no resiste', y eso no le movió un pelo, a nadie le movió un pelo, o sea, no está el tema ambiental, no es un tema de preocupación de este gobierno el tema ambiental.” (Referente ambientalista).

Vinculando estas ideas con las tipologías de naturaleza presentadas, podríamos entender que estos actores se corresponden con la visión "naturaleza bajo presión", al preocuparse por los impactos sobre el ambiente y los ecosistemas, cuestionar las externalidades positivas y denunciar las negativas. También despliegan una fuerte crítica a este modelo como propuesta de desarrollo y no solo enfocan las preocupaciones sobre las temáticas ambientales, sino también en la viabilidad económica, social y política del modelo. En sus discursos plantean problematizar los vínculos que, como sociedades, establecemos con el ambiente y el relacionamiento extractivista con la naturaleza.

Ahora bien, dentro del discurso de este grupo de entrevistados es posible identificar dos tipos de críticas al modelo celulósico: el primero apunta a cuestionar las externalidades positivas de este tipo de inversiones, como 
el hecho de estar en zonas francas, ${ }^{9}$ cuánto efectivamente del know how tecnológico queda en nuestro país, cuánto trabajo efectivamente generan, las concesiones y los prerrequisitos que exigen para su instalación, la dificultad de control ambiental, entre otros. El segundo nivel de crítica, más profundo, apunta a problematizar las relaciones sociedad/naturaleza, planteando las amenazas que este modelo productivo genera para las fuentes de agua y alimentarias, para la salud humana y ambiental y, en definitiva, para la reproducción de la vida. Esta segunda postura hace un llamado a respetar los derechos de la naturaleza y a cuestionar el modelo de desarrollo basado en la sobreexplotación de los bienes comunes.

\section{Reflexiones sobre los discursos y las vinculaciones sociedad/naturaleza}

En el análisis expuesto se describieron los discursos de algunos actores que, de diferentes maneras y grados de proximidad, están vinculados al modelo forestal-celulósico, especialmente a las plantas de celulosa. Se analizaron también las diferentes percepciones sobre los riesgos vinculados al modelo, atravesadas por las concepciones de naturaleza/ambiente y desarrollo que estos actores manejan.

Por un lado, encontramos el argumento de actores estatales y trabajadores de la producción celulósica, que legitima socialmente este tipo de emprendimientos en estrecha asociación con sus supuestos impactos positivos en las economías locales, que son presentadas como economías atrasadas, territorios vacíos o con escasas oportunidades. En esta postura, los beneficios de la inversión (mejores estándares de seguridad laboral y ambiental, mejores tecnologías para reducir la contaminación, revitalización de la economía, etcétera) justificarían la necesidad de asumir ciertos riesgos o aceptar ciertos niveles de contaminación. La definición de estos umbrales aceptables de riesgo parece estar en manos de determinado grupo de personas, en el marco de una lógica de legitimidad del conocimiento.

En estos argumentos predomina un discurso que refiere a una naturaleza fuerte y renovable, y en ellos parecen operar mecanismos de naturalización de la contaminación y una atención selectiva a los riesgos que puedan surgir. En este contexto, aparece con más fuerza la visión de la naturaleza como recurso para usar y manipular, es decir, una naturaleza separada (y al servicio) de la sociedad. Vale señalar que los sindicatos juegan un rol relevante, respaldando la idea de desarrollo y externalidades positivas. No obstante, tienen una mirada más abierta a la reflexión sobre la sustentabilidad ambiental de este modelo en el largo plazo, muy especialmente ante la posibilidad de la instalación de otras empresas de este tipo.

9 Áreas del territorio nacional cercadas y aisladas, con exenciones tributarias y demás beneficios. Ver Ley n. ${ }^{\circ} 19.566$. 
Por otro lado, los actores vinculados a las ONG ambientalistas plantearon un fuerte descontento con la gestión de los riesgos y las decisiones tomadas por los actores estatales, sintiéndose en muchos casos puestos a un lado en los debates. La crítica de estos actores no se limita a la producción de pasta de celulosa, sino que, en un sentido más amplio, cuestiona el modelo de desarrollo elegido para el país. Retomando las tipologías de naturaleza, estos actores comparten una visión de naturaleza sensible a los impactos de la acción humana y proponen una actitud cuidadosa hacia ella. También se pudo apreciar una valoración diferente de los impactos ambientales y un cuestionamiento más profundo en cuanto a los umbrales aceptables de riesgo.

Finalmente, es importante señalar que los talleres realizados como parte de la metodología de la investigación demostraron ser verdaderos espacios de diálogo, donde se pusieron en juego, en forma respetuosa, las diferentes posturas de los participantes, muchas veces muy disímiles.

Este trabajo, aún en desarrollo, representó un esfuerzo por contribuir al conocimiento sobre los discursos y percepciones referidos a los riesgos asociados a la producción de celulosa, especialmente en su vínculo con el ambiente y la naturaleza y los modelos de desarrollo. Asimismo, pretendió contribuir al debate sobre los vínculos que establecemos con la naturaleza, incorporando una perspectiva orientada a superar una visión productivista, para imaginar otro tipo de relacionamiento y estar abiertos a valorar en ese ambiente/naturaleza otros aspectos, más allá de su carácter de recurso. Se entiende que esta perspectiva permitiría un mejor análisis histórico coyuntural de la instalación de estas empresas en el Uruguay y de la participación de los diferentes sujetos, organizaciones e instituciones en estos procesos.

\section{Referencias bibliográficas}

Bartra, A. (2009). La gran crisis. Revista Venezolana de Economía y Ciencias Sociales, 15(2), pp. 191-202.

Beck, U. (1985). La sociedad del riesgo. Hacia una nueva modernidad. Buenos Aires: Paidós.

Carámbula, M. y D. Piñeiro (2006). La forestación en Uruguay: cambio demográfico y empleo en tres localidades. Agrociencia, 10(2), pp. 63-73.

Douglas, M. (1996). La aceptabilidad del riesgo según las ciencias sociales. Barcelona: Paidós.

Florit, P. (2013). La cadena forestal celulósica en Uruguay: contexto para la ampliación del puerto de La Paloma. Repercusiones de las inversiones forestales: la ampliación del puerto de La Paloma. Montevideo: CSIC, UDELAR. 
Gazzano, I. y M. Achkar (2013). La necesidad de redefinir ambiente en el debate científico actual. Gestión y Ambiente, 16(3), pp. 7-15.

Graña, F. (2010). "No hay nada que demostrar, Botnia va a contaminar". Un análisis del discurso de la Asamblea Ciudadana Ambiental de Gualeguaychú. Revista de Ciencias Sociales, XXIII(26), pp. 73-83.

Gudynas, E. (2013). Extracciones, extractivismos y extrahecciones. Un marco conceptual sobrelaapropiaciónderecursosnaturales.ObservatoriodelDesarrollo [en línea], 18.Disponible en:<http://ambiental.net/wp-content/uploads/2015/12/ GudynasApropiacionExtractivismoExtraheccionesOdeD2013.pdf $>$ [acceso 15 de marzo de 2020].

Gudynas, E. (2017). Neo-extractivismo y crisis civilizatoria. En: G. Ortega (coord.) (2017). América Latina: avanzando hacia la construcción de alternativas. Asunción: BASE-IS/FRL, pp. 29-54.

Ingold, T. (2000). The Perception of the Environment: Essays in livelihood, dwelling and skill. Londres: Routledge.

Juncal, A. y E. Fernández (2013). Valoración sobre el puerto multimodal de La Paloma. En: D. Piñeiro (coord.) (2013). Repercusiones de las inversiones forestales: la ampliación del puerto de La Paloma. Montevideo: CSIC, UDELAR, pp. 39-86.

Leff, E. (1998). Complejidad, racionalidad ambiental y diálogo de saberes. México: UNAM.

Machado Aráoz, H. (2014). Territorios y cuerpos en disputa: extractivismo minero y ecología política de las emociones. Intersticios, 8(1), pp. 56-71.

Nión, S. y V. Pereyra (2019). Sindicatos y Estado: discursos sobre riesgo en la producción de pasta de celulosa. En: V. Filardo (coord.) (2019). El Uruguay desde la Sociología XVII. Montevideo: DS, FCS, UDELAR, pp. 25-46.

Pérez Trento, N. (2020). Potencialidades y límites del enfoque (neo)extractivista en la dinámica de la acumulación de capital en la Argentina (1990-2016). Revista Izquierdas, 49, pp. 3974-3998.

Pucci F.; R. Levin; N. Trajtenberg y C. Bianchi (2006). La negociación de los umbrales aceptables de riesgo en la industria de la construcción uruguaya. Montevideo: DS, FCS, UDELAR. 
Quijano, A. (1992). Colonialidad y modernidad/racionalidad. En R. Blackburn y H. Bonilla (comps.) (1992). Los conquistados: 1492 y la población indígena de las Américas. Bogotá: FLACSO, pp. 11-20.

Sack, R. (1986). Human Territoriality: Its Theory and History. Cambridge: University Press.

Schwarz, M. y M. Thompson (1990). Divided we stand: re-defining politics, technology, and social choice. Pensilvania: Penn Press.

Scribano, A. (2012). Teorías Sociales del Sur: una mirada post-independentista. Córdoba: Estudios Sociológicos.

Svampa, M. (2012). Pensar el desarrollo desde América Latina [en línea]. Disponible en: <http://maristellasvampa.net/archivos/ensayo56.pdf $>$ [acceso 15 de marzo de 2020].

Svampa, M. y E. Viale (2014). Maldesarrollo. La Argentina del extractivismo y el despojo. Buenos Aires: Katz.

Tommasino, H.; A. Cortelezzi; M. Ackermann; L. Gorga; C. Petraglia; G. Souto; W. Annuziatto y D. Martin (2016). ¿Cómo impactó el crecimiento de la cadena forestal al agro y la economía uruguaya? En: OPYPA-MGAP (2016). Anuario OPYPA 2016. Montevideo: MGAP, pp. 503-526.

Uruguay XXI (2016). Informe del sector forestal en Uruguay. Inteligencia competitiva [en línea]. Montevideo: Uruguay XXI. Disponible en: $<$ http://www.camaramercantil.com.uy/uploads/cms_news_docs/SectorForestal-2016.pdf $>$ [acceso 15 de marzo de 2020].

Villegas, P. (2014). Amistades peligrosas: notas sobre movimientos sociales y gobiernos progresistas. En: M. A. Gandarillas (coord.) (2014). Extractivismos: nuevos contextos de dominación y resistencia. Cochabamba: CEDIB. 


\section{Anexo. Tabla 2. Referencia de siglas}

\begin{tabular}{cc}
\hline $\begin{array}{c}\text { DINAMA } \\
\text { MVOTMA }\end{array}$ & $\begin{array}{c}\text { Dirección Nacional de Medio Ambiente-Ministerio de Vivienda y Ordenamiento } \\
\text { Territorial }\end{array}$ \\
\hline MSP & Ministerio de Salud Publica \\
\hline IGTSS-MTSS & $\begin{array}{c}\text { Inspección General de Trabajo y Seguridad Social-Ministerio de Trabajo y } \\
\text { Seguridad Social }\end{array}$ \\
\hline MIEM & Ministerio de Industria, Energía y Minería \\
\hline AFPAPEL & Asociación de Fabricantes de Papel \\
\hline FOPCU & Federación Obreros Papeleros Cartoneros del Uruguay \\
\hline REDES & Redes Amigos de la Tierra \\
\hline UDELAR & Universidad de la Republica \\
\hline MDP & UPM-Kymmene del Plata \\
\hline UPM & Facultad de Química. Universidad de la Republica \\
\hline FO & Facultad de Derecho, UDELAR \\
\hline FDER & Facultad de Ciencias, UDELAR \\
\hline FCIEN & Facultad de la Información y la Comunicación, UDELAR \\
\hline FIC & Facultad de Arquitectura, Diseño y Urbanismo, UDELAR \\
\hline FADU & Facultad de Medicina, UDELAR \\
\hline FMED & \\
\hline &
\end{tabular}

\section{Contribución de autoría}

Este trabajo fue realizado en su totalidad por Valentina Pereyra Ceretta. 